\title{
Topological protection from exceptional points in Weyl and nodal-line semimetals
}

\author{
J. González and R. A. Molina \\ Instituto de Estructura de la Materia, Consejo Superior de Investigaciones Científicas, Serrano 123, 28006 Madrid, Spain
}

(Received 3 March 2017; revised manuscript received 8 May 2017; published 31 July 2017)

\begin{abstract}
We investigate the topological protection of surface states in Weyl and nodal-line semimetals by characterizing them as evanescent states when the band structure is extended to complex momenta. We find in this way a sequence of exceptional points—-that is, branch points with zero energy in the complex spectrum—allowing us to identify the set of surface states with complex momentum signaling the decay into the 3D semimetal. From this point of view, Weyl and nodal-line semimetals can be classified in two types depending on the way surface states decay. Type A semimetals have surface states with smaller penetration length and oscillating decay while type B semimetals have longer simple exponential decays. The difference between both types reflects in the way the branch cuts in the spectrum accommodate in the complex plane. The stability of the surface states stems in this approach from the complex structure that develops around the exceptional points, with a topological protection which is based on the fact that the branch cuts cannot be closed by small perturbations. We check this property when nodal-line semimetals are placed under circularly polarized light, where we observe that the exceptional points survive the effect of such a perturbation, though appropriate boundary conditions for zero-energy surface states cannot be satisfied in general due to the breakdown of time-reversal invariance by the radiation field.
\end{abstract}

DOI: 10.1103/PhysRevB.96.045437

\section{INTRODUCTION}

Topological materials have become a primary target of research in the last few years due to their exceptional properties with high potential for applications [1,2]. The bulk-edge correspondence and the topological protection of edge states is on the basis of the interesting properties of systems with topologically nontrivial band structure. In topological insulators, the quantity controlling the topological protection is the inverted gap between bands arising from the crystal structure of the material. A perturbation must be strong enough to close the gap in order to destroy the edge states of the material.

In band theory, a semimetal is usually defined as a material with no gap in the band structure but with a very small overlap between the valence and the conduction band, resulting in a small density of states around the Fermi energy. A typical example is bismuth which has intermediate properties between an insulator and a metal [3]. In topological band theory, however, it is customary to work with a more strict definition by which a semimetal is a material with no gap but also no Fermi surface, so the conduction and valence bands touch only at isolated points. The semimetal so defined is really a distinct phase intermediate between insulators and metals, the most famous example being graphene [4]. Although the Dirac nodes in the 2D Brillouin zone of graphene are not topologically protected, in three dimensions we have the last additions to the family of topological materials, which include Dirac and Weyl semimetals with isolated Dirac or Weyl nodes in the band structure [5-8], and the nodal-line semimetals with a continuous line of nodes in the Brillouin zone [9].

The surface states corresponding to these topological semimetals lie on constant energy contours which do not form in general closed curves. In the case of the Weyl and Dirac semimetals, the surface states lead to the celebrated Fermi arcs joining the projection of the nodes onto the given surface. For nodal-line semimetals, the surface states form the so-called drumhead within the nodal circle [9-11]. In the Weyl semimetals, such states are topologically protected and
Chern numbers can be defined in the planes lying between Weyl nodes [12,13]. The physical quantity measuring this protection is the separation of the nodes in momentum space. The situation is less clear for Dirac semimetals in which a simple application of bulk-surface correspondence does not provide with an answer to whether or not the surface states are topologically protected. Recent works have discussed the stability of the surface states in 3D semimetals [14-16]. In this regard, an alternative description of the topological protection of surface states in 3D topological semimetals is very desirable. Here, we provide such a description by extending the band structure in momentum space to complex momenta.

The convenience of taking complex values of the momentum is motivated by the search of evanescent states in the $3 \mathrm{D}$ semimetals. When the momentum is promoted to a complex quantity, the Hamiltonian of the system becomes non-Hermitian, but it is still possible to find exceptional points in the spectrum, that is, branch points where the imaginary part of the eigenvalue vanishes. This allows one to identify the set of evanescent states with complex momentum that signals the decay into the 3D semimetal. Such states are endowed with topological protection, which arises naturally from the fact they are attached to branch cuts that cannot be removed under small perturbations. This description based on the identification between evanescent states and exceptional points was pioneered in a previous work describing surface states in Weyl semimetals in the presence of circularly polarized light [17]. Recently, rings of exceptional points have been also found in dissipative systems which are based on Weyl semimetals [18].

When looking for evanescent states in the spectrum of the 3D semimetals, we have found that these can be classified in two different groups, depending on the pattern of the branch cuts in the plane of complex momentum. Thus, there is a class of $3 \mathrm{D}$ semimetals, that we denote as type $\mathrm{A}$, where most part of the branch cuts run in parallel crossing the real axis. The corresponding exceptional points form then quartets belonging to the same branch of the spectrum, with each member of a 
quartet in a different quadrant of the complex plane. In the other class, that we denote as type B, all the branch cuts can be disposed instead along the imaginary axis, lacking the nontrivial realization of symmetry found in the type A class. From the physical point of view, this introduces also an important difference between the two classes, as the quartets found in the type A semimetals provide a higher degree of topological protection, quantified in terms of a larger length of the branch cuts and a much smaller penetration length of the evanescent states in the 3D semimetal.

Note that this classification has a different origin than the classification into type I and type II Weyl semimetals, that depends on whether the density of states vanishes at the nodal points or has some extra contributions due to the tilting of the Weyl cones $[19,20]$. Although we have only explored a model describing a type I Weyl semimetal in this work, we expect our classification to be supplementary to the classification into type I and type II Weyl semimetals, that is, we expect to be type A semimetals of type I and type II and type B semimetals also of both type I and II.

The stability of the surface states stems in our approach from the complex structure that develops for complex momentum, where different bands can be seen as different branches of the Riemann surface giving the spectrum. In this framework, a pair of exceptional points and the respective evanescent states can be only annihilated by merging the branch points, provided they lie in the same branch of the spectrum. This picture allows us to establish a connection with the usual account of the topological protection of surface states, which requires making a $2 \mathrm{D}$ projection of the $3 \mathrm{D}$ band structure to open a gap in the spectrum. In our approach a gap also exists, but this is now seen as the separation opened between different branches along a branch cut in the complex spectrum. Such a gap can be closed only as long as the exceptional points at the two ends of the branch cut are made to coalesce, which provides an alternative understanding of the topological protection in the plane of complex momentum.

In this paper, we apply the complex structure developed around the exceptional points to investigate the stability of the surface states in 3D Weyl and nodal-line semimetals. We are going to see that this approach provides a very robust picture of the evanescent states in these systems. This will be checked in particular in the case of the nodal-line semimetals under circularly polarized light, which is an instructive example since that may not be in general a small perturbation of the semimetal. We will see that the exceptional points survive indeed the effect of the electromagnetic field, though appropriate boundary conditions for surface states cannot be satisfied in general due to the breakdown of time-reversal invariance by the radiation field.

The paper is organized as follows. In Sec. II, we consider Weyl semimetals and their surface states on the light of our approach to look for evanescent states with complex momentum. Then, in Sec. III, we consider nodal-line semimetals, their bulk and their surface states in cylindrical coordinates which are well-suited to the problem. In Sec. IV, we use the same approach to study the behavior of evanescent states in the case of a periodic perturbation of the nodal-line semimetal by circularly polarized light. We finish with some conclusions of our study in Sec. V.

\section{WEYL SEMIMETAL}

We consider a simple model for a Weyl semimetal with Hamiltonian

$$
H_{\mathrm{w}}=\left(m_{0}+m_{1} \nabla^{2}\right) \sigma_{z}-i v \partial_{z} \sigma_{x}-i v \partial_{y} \sigma_{y} .
$$

The energy-momentum dispersion as a function of the 3D momentum $\mathbf{k}$ is given then by

$$
\varepsilon= \pm \sqrt{\left(m_{0}-m_{1} \mathbf{k}^{2}\right)^{2}+v^{2} k_{y}^{2}+v^{2} k_{z}^{2}} .
$$

It turns out that the valence and conduction bands touch at Weyl points located in the line $k_{y}=k_{z}=0$ with

$$
k_{x}= \pm \sqrt{\frac{m_{0}}{m_{1}}} .
$$

In this model, we may look for surface states characterized by wave functions decaying for instance in the $z$ direction as

$$
\phi(x, y, z) \sim e^{i k_{z} z} e^{-\alpha z} f(x, y),
$$

with $\alpha>0$ corresponding to the inverse of the penetration length. The action of the Hamiltonian becomes particularly simple if we concentrate on the set of states

$$
\begin{aligned}
& \phi_{k_{x}, k_{z},+}(x, y, z)=e^{i k_{x} x} e^{i k_{z} z} e^{-\alpha z}|+\rangle, \\
& \phi_{k_{x}, k_{z},-}(x, y, z)=e^{i k_{x} x} e^{i k_{z} z} e^{-\alpha z}|-\rangle
\end{aligned}
$$

with the spinor part corresponding to the eigenvectors of $\sigma_{y}$,

$$
|+\rangle=\left(\begin{array}{l}
1 \\
i
\end{array}\right), \quad|-\rangle=\left(\begin{array}{c}
1 \\
-i
\end{array}\right) \text {. }
$$

We get in this way

$$
\begin{aligned}
H_{\mathrm{w}} \phi_{k_{x}, k_{z},+}= & \left(m_{0}-m_{1}\left(k_{x}^{2}+k_{z}^{2}-\alpha^{2}+2 i k_{z} \alpha\right)\right. \\
& \left.+i v\left(k_{z}+i \alpha\right)\right) \phi_{k_{x}, k_{z},-} \\
H_{\mathrm{w}} \phi_{k_{x}, k_{z},-}= & \left(m_{0}-m_{1}\left(k_{x}^{2}+k_{z}^{2}-\alpha^{2}+2 i k_{z} \alpha\right)\right. \\
& \left.-i v\left(k_{z}+i \alpha\right)\right) \phi_{k_{x}, k_{z},+} .
\end{aligned}
$$

Then we can identify a collection of zero-energy modes in the set of states $\left\{\phi_{k_{x}, k_{z},+}\right\}$ by canceling out the right-hand side of Eq. (8) (assuming that $m_{0}>0, m_{1}>0, v>0$ ). This leads to two types of solutions, either

$$
\begin{gathered}
\alpha=\frac{v}{2 m_{1}}, \\
k_{z}= \pm \sqrt{\frac{m_{0}}{m_{1}}-k_{x}^{2}-\alpha^{2}}
\end{gathered}
$$

or

$$
\begin{gathered}
\alpha=\frac{v \pm \sqrt{v^{2}-4 m_{1}\left(m_{0}-m_{1} k_{x}^{2}\right)}}{2 m_{1}}, \\
k_{z}=0 .
\end{gathered}
$$

For a given $k_{x}$, two independent states with $\alpha \geqslant 0$ exist as long as $k_{x}^{2} \leqslant m_{0} / m_{1}$. We note that the actual wave function corresponding to a surface state must be a linear combination of the solutions with + and - signs [in either (10) and (11) 
or (12) and (13)] in order to fulfill appropriate boundary conditions (for example, in a semi-infinite plane, making the wave function to vanish at $z=0$ ). This collection of evanescent states maps therefore the celebrated Fermi arcs with $\left|k_{x}\right| \leqslant \sqrt{m_{0} / m_{1}}$, which join the projection of the Weyl points onto a given surface of the semimetal.

The solution corresponding to Eqs. (10) and (11) is valid when $4 m_{1} m_{0}>v^{2}$. In this case, there is, however, a portion of the Fermi arcs, closer to the endpoints, where the states change to the form given by Eqs. (12) and (13). Thus, we identify a class of Weyl semimetals that we denote as type A (corresponding in our model to $4 m_{1} m_{0}>v^{2}$ ) in which two different kinds of states coexist in the Fermi arcs, with oscillatory decay along the $z$ direction in the range

$$
0 \leqslant\left|k_{x}\right|<\sqrt{\frac{m_{0}}{m_{1}}-\frac{v^{2}}{4 m_{1}^{2}}}
$$

and pure exponential decay in the range

$$
\sqrt{\frac{m_{0}}{m_{1}}-\frac{v^{2}}{4 m_{1}^{2}}} \leqslant\left|k_{x}\right|<\sqrt{\frac{m_{0}}{m_{1}}} .
$$

In this regime, we observe that the set of states with oscillatory decay shrinks progressively as $v$ grows, until they disappear at $v^{2}=4 m_{1} m_{0}$. This variable proportion of evanescent states with oscillatory decay and pure exponential decay is illustrated in Fig. 1 in two different cases with $4 m_{1} m_{0} \gg v^{2}$ and $4 m_{1} m_{0} \approx v^{2}$. The plots represent the lowest eigenvalue of $H_{\mathrm{w}}$ when it is diagonalized in the space spanned by the basis (5) and (6). The Hamiltonian becomes non-Hermitian as the momentum in the $z$ direction is extended to complex values $k_{z}+i \alpha$, but one can still look for zero-energy modes in the spectrum. In Figs. 1(a)-1(d), one can clearly see the lines in which both the real and the imaginary part of the eigenvalue vanish, mapping the different zero-energy evanescent states along the Fermi arc with $0 \leqslant k_{x} \leqslant \sqrt{m_{0} / m_{1}}$.

Moreover, we find a complementary class of Weyl semimetals that we will refer to as type B (corresponding in our description to $4 m_{1} m_{0}<v^{2}$ ) characterized by having Fermi arcs where all the states have pure exponential decay. Their wave function has in general a dependence on the $z$ coordinate given by

$$
\phi \sim c_{1} e^{-\alpha_{+} z}+c_{2} e^{-\alpha_{-} z},
$$

$\alpha_{ \pm}$being the two solutions in (12). The smallest of the two values of $\alpha$ dictates the inverse penetration length, which may run from 0 up to $v / 2 m_{1}$. This leads therefore to a slower decay along the $z$ direction in comparison to the evanescent states with oscillatory behavior in the type A semimetals.

This distinction between the two mentioned classes is relevant since the evanescent states with oscillatory decay have a different phenomenology than the evanescent states with pure exponential decay. This can be more easily realized when we consider a slab of finite width $W$, with two opposite faces of the material oriented perpendicularly with respect to the $z$ axis. If we have evanescent modes with opposite values of $k_{z}$, they can be superposed pairwise to form eigenstates with a dependence on the $z$ variable:

$$
\phi \sim \sin \left(k_{z} z\right) e^{-\alpha z} .
$$

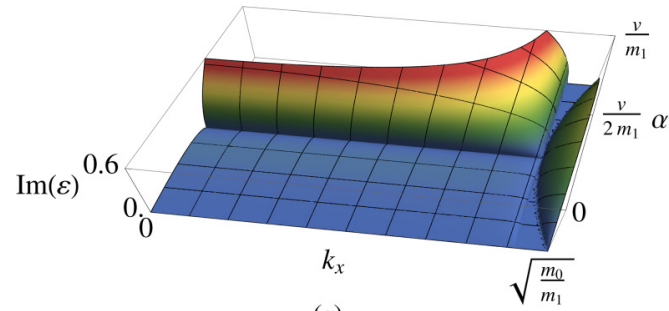

(a)

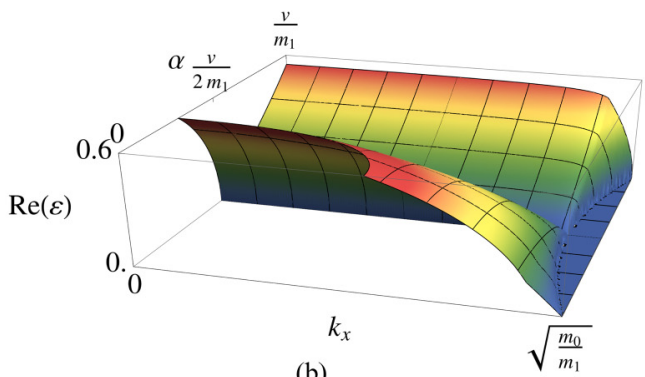

(b)

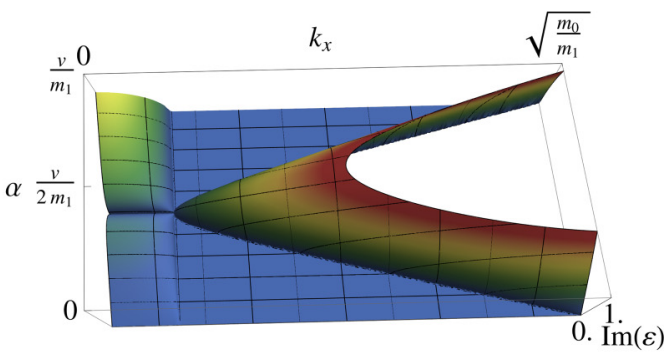

(c)

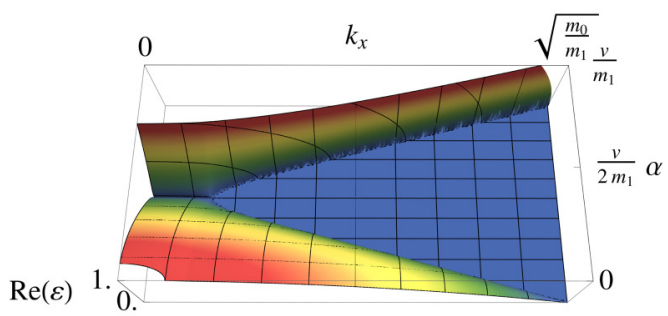

(d)

FIG. 1. [(a) and (b)] Representation of the absolute value of the imaginary part and the real part of the lowest eigenvalue of $H_{\mathrm{w}}$ for states with complex momentum $k_{z}+i \alpha$ along the Fermi arc with $0 \leqslant$ $k_{x} \leqslant \sqrt{m_{0} / m_{1}}$, for $m_{0}=1.0 \mathrm{eV}, m_{1} / a^{2}=1.0 \mathrm{eV}$, and $v / a=0.8 \mathrm{eV}$ ( $a$ being a microscopic length scale in the model). The energy $\varepsilon$ is measured in units of eV. [(c) and (d)] Similar representation as in (a) and (b) but for $m_{0}=1.0 \mathrm{eV}, m_{1} / a^{2}=1.0 \mathrm{eV}$, and $v / a=1.98 \mathrm{eV}$. The plots map the distinctive zero-energy modes with constant $\alpha$ (corresponding to states with oscillatory decay) and the bifurcation into two zero-energy branches with variable $\alpha$ (representing the states with pure exponential decay).

Then, it becomes possible to build surface states decaying along the $z$ direction with the right boundary conditions (vanishing at the two faces of the material, say at $z=0$ and $z=W$ ) in a way which does not require to know about the surface states localized near the other face of the material (at $z=W$ ). This construction cannot be done, however, with the evanescent modes having pure exponential decay. In that case, eigenstates with a dependence like that in (16) may be chosen 


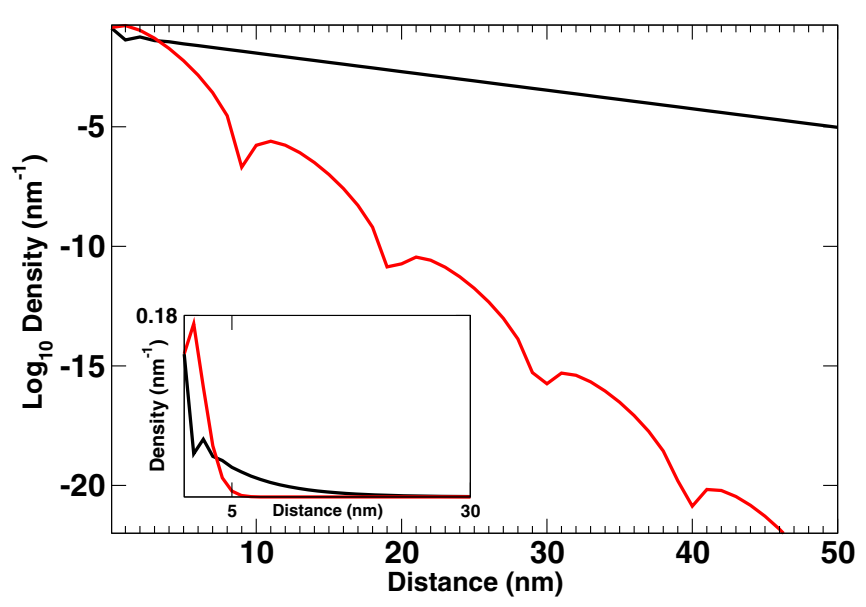

FIG. 2. Probability density in logarithmic scale across the $z$ direction of surface states in the Fermi arcs with $k_{x}=0$. We compare two different cases, one with some oscillatory dependence in the $z$ direction corresponding to a type A Weyl semimetal (red line, $m_{0}=$ $0.35 \mathrm{eV}, m_{1}=1.0 \mathrm{eV} \mathrm{nm}^{2}$, and $v=1.0 \mathrm{eV} \mathrm{nm}$ ) and another with pure exponential decay corresponding to a type B Weyl semimetal (black line, $m_{0}=0.35 \mathrm{eV}, m_{1}=1.0 \mathrm{eV} \mathrm{nm}^{2}$, and $v=4.0 \mathrm{eV} \mathrm{nm}$ ). In the inset, the same figure is shown in normal scale.

to vanish at $z=0$, but it cannot be avoided some leaking of their amplitude to the opposite face at $z=W$. In other words, evanescent states with pure exponential decay may still lead to proper surface states in a slab, but they require necessarily the mixing of modes localized at opposite faces of the material.

In Fig. 2, we show numerical calculations for an equivalent tight-binding Hamiltonian in a slab of width $W=1000 \mathrm{~nm}$ in the $z$ direction, for two different examples pertaining to type A and type B semimetals. The appearance of the band structure is very similar in the two regimes, with the Fermi arcs joining the projection of the Weyl nodes. However, in the type B regime $\left(4 m_{1} m_{0}<v^{2}\right)$ the Fermi arc states have a penetration length $(1 / \alpha)$ that increases with the value of $\left|k_{x}\right|$ until it diverges at the projection of the Weyl nodes. In the type A regime $\left(4 m_{1} m_{0}>v^{2}\right)$, the states with $k_{x}^{2}<m_{0} / m_{1}-v^{2} / 4 m_{1}^{2}$ have a very small penetration length $2 m_{1} / v$, which would be typically of the order of a few unit cells in real materials. However, the probability density oscillates as it decays. For larger values of $k_{x}^{2}$, the penetration length grows until it diverges again at the projection of the Weyl nodes, and the wave function for $z$ is just a decaying exponential without oscillations.

The different character of the states with oscillatory decay and those with pure exponential decay has also a reflection in the behavior of their energy in a slab. The surface states of the type B semimetals have always nonvanishing energy (measured with respect to that of the nodal points) as they are built from the hybridization of evanescent waves at two opposite faces of the slab. On the other hand, the energy of the surface states of type A semimetals vanishes when the condition of resonance is met along the finite dimension of the slab, which implies a nonmonotonous dependence on the width $W$ as shown in Fig. 3. We note that this also leads in general to different orders of magnitude when comparing the energies of the surface states in type A and type B semimetals, as seen in the figure.

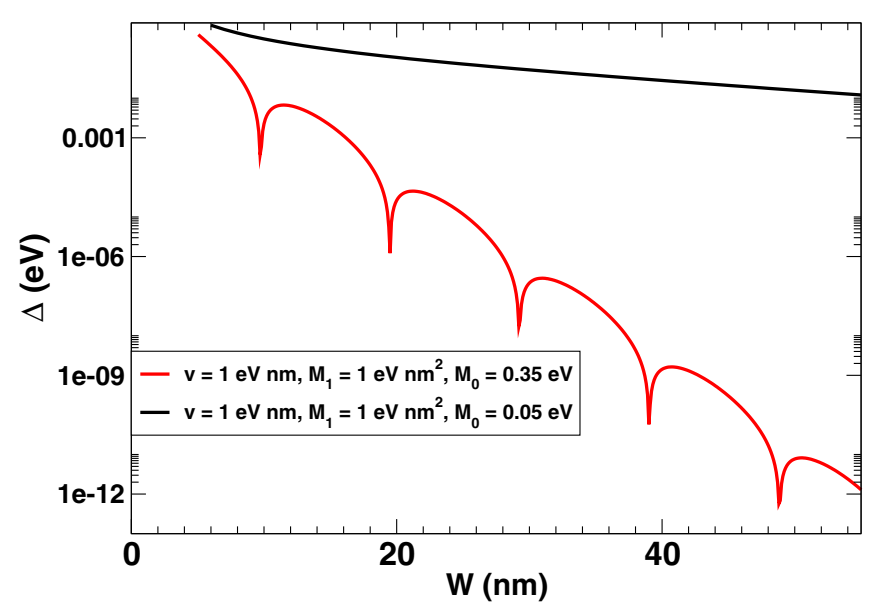

FIG. 3. Energy in log scale of surface states at the midpoint of the Fermi $\operatorname{arc}\left(k_{x}=0\right.$ in our model) as a function of the width $W$ in a slab geometry, comparing the behavior for a type A semimetal (red line, $m_{0}=0.35 \mathrm{eV}, m_{1}=1.0 \mathrm{eV} \mathrm{nm}^{2}$, and $v=1.0 \mathrm{eV} \mathrm{nm}$ ) and for a type B semimetal (black line, $m_{0}=0.05 \mathrm{eV}, m_{1}=1.0 \mathrm{eV} \mathrm{nm}{ }^{2}$, and $v=1.0 \mathrm{eV} \mathrm{nm}$ ).

Within this approach, we can also analyze the role that the evanescent states play in the spectrum of the Hamiltonian $H_{\mathrm{w}}$, as this becomes a non-Hermitian operator acting on the basis (5) and (6). In the subspace spanned by this set of states, the eigenvalues of $H_{\mathrm{w}}$ turn out to be according to (8) and (9):

$$
\lambda= \pm \sqrt{\left(m_{0}-m_{1}\left(k_{x}^{2}+\left(k_{z}+i \alpha\right)^{2}\right)\right)^{2}+v^{2}\left(k_{z}+i \alpha\right)^{2}} .
$$

It can be seen that the particular values satisfying the zeromode conditions (10), (11) and (12), (13) correspond to branch points in the complex spectrum of $H_{\mathrm{W}}$, both for $4 m_{0} m_{1}>v^{2}$ and $4 m_{0} m_{1}<v^{2}$. We find therefore that the evanescent states we have identified correspond to so-called exceptional points [21,22] in the spectrum of $H_{\mathrm{w}}$, when this is mapped as a function of the complex momentum $k_{z}+i \alpha$. This highlights that there is a complex structure behind the surface states of the Weyl semimetals, which has important implications for their stability.

The structure of the branch cuts in the complex plane $k_{z}+i \alpha$ is different however, depending on whether we consider the type $\mathrm{A}$ regime $\left(4 m_{0} m_{1}>v^{2}\right)$ or the type $\mathrm{B}$ regime $\left(4 m_{0} m_{1}<v^{2}\right)$ of the Weyl semimetal. In the first case, the states in the Fermi arcs with $k_{x}^{2}<m_{0} / m_{1}-v^{2} / 4 m_{1}^{2}$ correspond to branch points that are away from the imaginary axis, as represented in Fig. 4, where it can be observed the branch cuts that develop from the location of the zero-energy modes. The branch cuts fall eventually into the imaginary axis for $k_{x}^{2}>m_{0} / m_{1}-v^{2} / 4 m_{1}^{2}$, where we know that the evanescent states must be in accordance with (12) and (13). In the type $B$ regime, however, the branch cuts are found in the imaginary axis for all the states in the Fermi arcs, with a typical structure represented in Fig. 5.

The different structure of the branch cuts in the type A and type B regimes of the Weyl semimetal can be actually ascribed to a different realization of the symmetries of the Hamiltonian (1). This is in particular invariant under the spatial inversion, which can be represented as an operator $I$ with an action on 


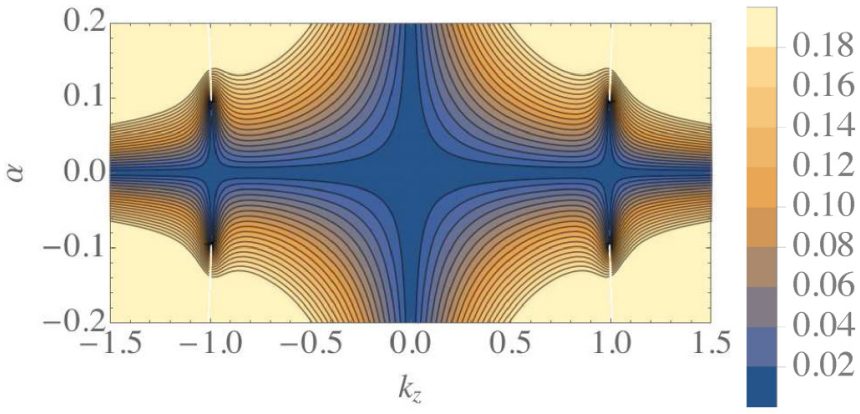

(a)

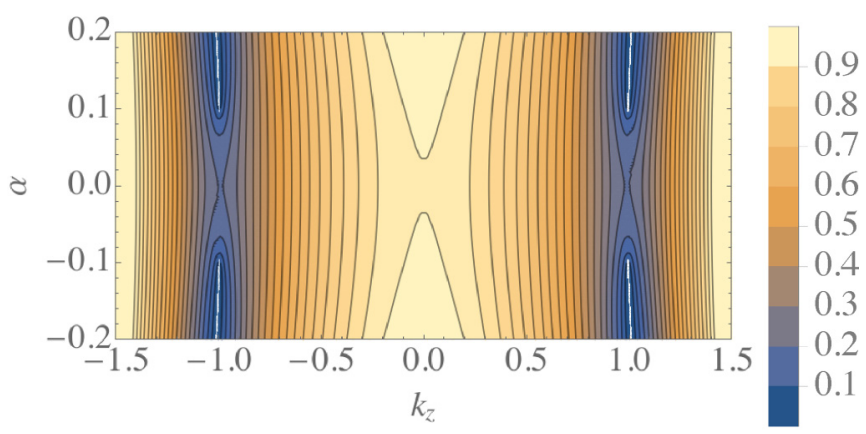

(b)

FIG. 4. Contour plots of the absolute value of the imaginary part (a) and the real part (b) of the eigenvalue in (18) for $k_{x}=0$ and $m_{0}=$ $1.0 \mathrm{eV}, m_{1} / a^{2}=1.0 \mathrm{eV}$, and $v / a=0.2 \mathrm{eV}, a$ being a microscopic length scale in the model. $k_{z}$ and $\alpha$ are measured in units of $a^{-1}$ and the energy is in units of eV.

\section{$\begin{array}{lllllllll}0.1 & 0.2 & 0.3 & 0.4 & 0.5 & 0.6 & 0.7 & 0.8 & 0.9\end{array}$}

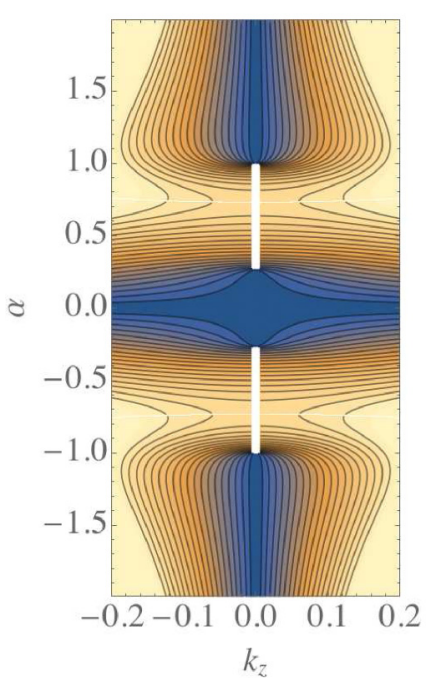

(a)

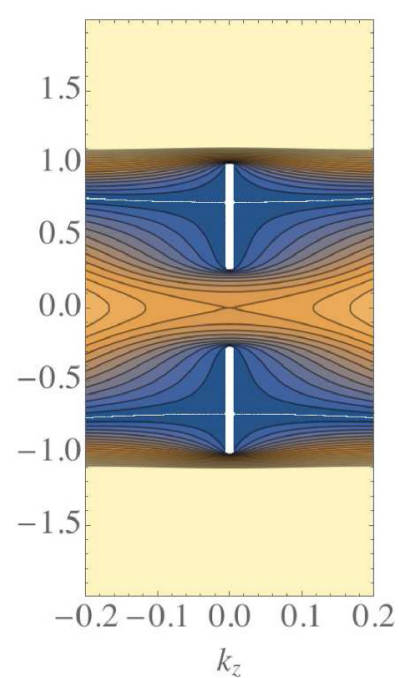

(b)
FIG. 5. Contour plots of the absolute value of the imaginary part (a) and the real part (b) of the eigenvalue in (18) for $k_{x}=0$ and $m_{0}=$ $0.5 \mathrm{eV}, m_{1} / a^{2}=1.9 \mathrm{eV}$, and $v / a=2.4 \mathrm{eV}, a$ being a microscopic length scale in the model. $k_{z}$ and $\alpha$ are measured in units of $a^{-1}$ and the energy is in units of eV. the states $\phi(x, y, z)$ given by

$$
I: \phi(x, y, z) \rightarrow \sigma_{z} \phi(-x,-y,-z) .
$$

The Hamiltonian (1) has moreover an enlarged symmetry when the dynamics is constrained to states that do not depend on the $y$ coordinate, as in the above discussion. Then $H_{\mathrm{w}}$ becomes invariant under a transformation $T$ that acts like time-reversal invariance, given in terms of the operation of complex conjugation $K$ as

$$
T: \phi(x, y, z) \rightarrow K \sigma_{z} \phi(x, y, z) .
$$

Thus, in the structure represented in Fig. 4 for the type A regime of the Weyl semimetal, the two branch points related by the inversion of the complex momentum $k_{z}+i \alpha$ correspond to states that are mapped onto each other by the action of $I$. On the other hand, the transformation $T$ is realized in the complex plane as the inversion $k_{z} \rightarrow-k_{z}$. This accounts for the fact that the same state $\phi(x, y, z)$ (with $y=$ const.) is found at the two branch points related by such an inversion of the momentum $k_{z}$.

The fact that one finds the same evanescent state at branch points with momenta $k_{z}$ and $-k_{z}$ is important from the point of view of the stability of the surface states. As already mentioned, two independent states $\phi(x, y, z)$ are needed in order to build a surface state that may vanish on the boundary of the system, say for instance on the plane $z=0$. This requirement can be satisfied in the type A regime of the Weyl semimetal from the symmetry enforced by the $T$ operation. Then, the surface states with $k_{z} \neq 0$ turn out to be protected due to the particular structure of the branch cuts, which cannot be undone unless the branch points are made to coalesce in pairs. Such a kind of topological protection holds also in the type B regime of the Weyl semimetal, with evanescent states which correspond to branch points with more variable separation along the imaginary axis and can merge in general under weaker perturbations.

We find that the exceptional points endow in general the surface states with topological protection, as the nontrivial topology of the branch cuts cannot be modified with small perturbations. The distinctive feature of the branch points is that one needs to turn twice around them to return to the original point in the spectrum, as observed in the $3 \mathrm{D}$ plot of the complex dispersion in Fig. 6(a). This has a reflection in the fact that the complex eigenvalues as well as the eigenfunctions get a Berry phase of $\pi$ after going around any of the branch points.

Anyhow, the mathematical basis of the topological protection lies in our approach in the complex structure of the eigenvalues, rather than in the behavior of the eigenfunctions in the $\left(k_{z}, \alpha\right)$ plane. As illustrated in Fig. 6(a) for the case of a type B Weyl semimetal, there are two branch cuts in each of the subbands of the spectrum (labeled here by the momentum $k_{x}$ ). In order to make a closed orbit in the complex spectrum, one needs to enclose two branch points as sketched in Fig. 6(b). Looking at the 3D plot, it becomes clear that this creates noncontractible loops, making manifest that the Riemann sheet does not have the topology of a sphere. Indeed, it is known that a Riemann sheet with two branch cuts of square-root type has the topology of a torus (once the space is compactified by identifying the points at infinity). This is consistent with the existence of two independent noncontractible orbits in the 3D 


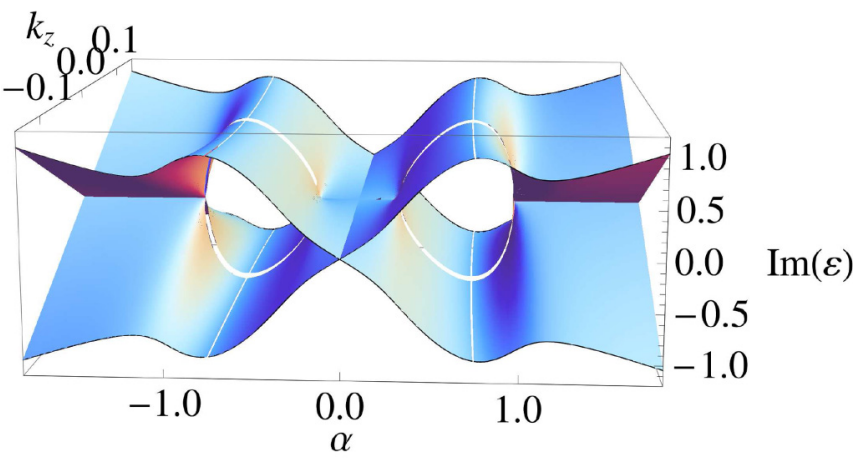

(a)

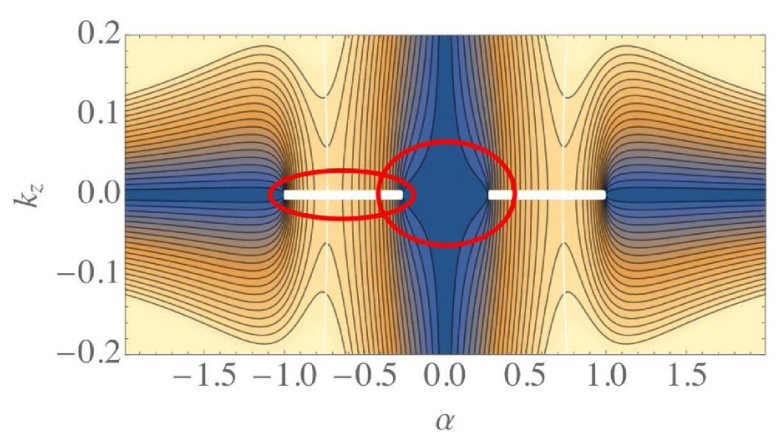

(b)

FIG. 6. (a) 3D plot of the imaginary part of the eigenvalue in (18) for $k_{x}=0$ and the same parameters as in Fig. $5\left(m_{0}=0.5 \mathrm{eV}\right.$, $m_{1} / a^{2}=1.9 \mathrm{eV}$, and $\left.v / a=2.4 \mathrm{eV}\right) . k_{z}$ and $\alpha$ are measured in units of $a^{-1}$ and the energy is in units of eV. (b) Contour plot of the absolute value of the imaginary part of the eigenvalue in (18) for the same parameters as in (a) and with the same units as in Fig. 5. The red circles stand for the projection of two noncontractible loops around the branch points in the spectrum.

plot of Fig. 6(a), which correspond actually to the two cycles of the torus.

Although the nontrivial topology of the spectrum is best visualized in the 3D plot for the type B Weyl semimetal, similar considerations apply to the type A class with the branch cuts shown in Figs. 4(a) and 4(b). As a generic feature, the branch cuts are tied to the existence of a gap in the spectrum, which corresponds to the distance between the two branches of the Riemann sheet. We see that this gap is part of a more involved structure in our approach, which unveils the nontrivial topology of the spectrum. As in the conventional description of the surface states in a Weyl semimetal, these are protected as long as the gap persists in the spectrum, which is enforced here by the impossibility to change its toroidal topology by means of smooth perturbations.

\section{NODAL-LINE SEMIMETAL}

\section{A. Bulk states}

Our starting point is a model of nodal-line semimetal with a Hamiltonian

$$
H_{\mathrm{NL}}=\left(m_{0}+m_{1} \nabla^{2}\right) \sigma_{z}-i v \partial_{z} \sigma_{x} .
$$

In terms of the 3D momentum $\mathbf{k}$, the eigenvalues of (21) are given by

$$
\varepsilon= \pm \sqrt{\left(m_{0}-m_{1} \mathbf{k}^{2}\right)^{2}+v^{2} k_{z}^{2}}
$$

This model has then a line of nodes in the plane $k_{z}=0$, given by the circular set

$$
k_{x}^{2}+k_{y}^{2}=\frac{m_{0}}{m_{1}} .
$$

When looking for energy eigenstates, we can concentrate on modes with well-defined angular momentum, with wave function $\psi$ such that in polar coordinates $(r, \theta, z)$

$$
\psi(r, \theta, z) \sim e^{i k_{z} z} e^{i m \theta} f(r) .
$$

The spectrum can be obtained then by solving the eigenvalue problem

$$
\begin{gathered}
{\left[m_{0}+m_{1}\left(\frac{1}{r} \frac{\partial}{\partial r}\left(r \frac{\partial}{\partial r}\right)-\frac{m^{2}}{r^{2}}-k_{z}^{2}\right)\right]} \\
\times \sigma_{z} \psi_{k_{z}}^{(m)}+v k_{z} \sigma_{x} \psi_{k_{z}}^{(m)}=\varepsilon \psi_{k_{z}}^{(m)} .
\end{gathered}
$$

In the case of bulk zero-energy modes, we see from (25) that they can be expressed in terms of Bessel functions $J_{m}$ as

$$
\begin{aligned}
& \psi_{0,+}^{(m)}(r, \theta, z)=e^{i m \theta} J_{m}(k r)|u\rangle, \\
& \psi_{0,-}^{(m)}(r, \theta, z)=e^{i m \theta} J_{m}(k r)|d\rangle
\end{aligned}
$$

with $k=\sqrt{m_{0} / m_{1}}$ and the spinor part given by the eigenvectors of $\sigma_{z}$ :

$$
|u\rangle=\left(\begin{array}{l}
1 \\
0
\end{array}\right), \quad|d\rangle=\left(\begin{array}{l}
0 \\
1
\end{array}\right) .
$$

It turns out that, in this particular representation, the states corresponding to the nodal line are labeled by the integer values $m$ of the projection of the angular momentum along the $z$ direction.

\section{B. Surface states}

We are interested in surface states that take the form of evanescent waves localized at the boundary of the $3 \mathrm{D}$ semimetal. It can be easily seen that there is a huge set of these states characterized by the evanescence in the $z$ direction, with wave function $\chi$ decaying as

$$
\chi(r, \theta, z) \sim e^{i k_{z} z} e^{-\alpha z} e^{i m \theta} f(r) .
$$

The zero-energy modes have to correspond in particular to solutions of the equation

$$
\begin{aligned}
& {\left[m_{0}+m_{1}\left(\frac{1}{r} \frac{\partial}{\partial r}\left(r \frac{\partial}{\partial r}\right)-\frac{m^{2}}{r^{2}}-k_{z}^{2}+\alpha^{2}-2 i k_{z} \alpha\right)\right]} \\
& \times \sigma_{z} \chi_{k_{z}}^{(m)}+v\left(k_{z}+i \alpha\right) \sigma_{x} \chi_{k_{z}}^{(m)}=0 .
\end{aligned}
$$

First of all, the imaginary terms must cancel out in (30). This means that the solutions must be necessarily proportional to the eigenvectors $| \pm\rangle$ of $\sigma_{y}$ given in (7). Taking the first spinor, one obtains the constraint

$$
-2 i m_{1} k_{z} \alpha+i v k_{z}=0,
$$


which leads to either

$$
\alpha=\frac{v}{2 m_{1}}
$$

or

$$
k_{z}=0
$$

Similarly to the case of the Weyl semimetals, the condition (32) gives rise to evanescent states with oscillatory decay while the condition (33) results in an exponential decay without oscillations. If we choose otherwise the second spinor in (7), that changes the sign of the last term in the left-hand side of (30), which prevents the existence of evanescent states with $k_{z} \neq 0$ (for $v>0, m_{1}>0$ ) and does not allow either to find solutions with $\alpha>0$ when $k_{z}=0$ (as we see in what follows).

Taking the value of $\alpha$ in (32), it turns out that the evanescent states are given by the solutions of Eq. (30):

$$
\chi_{k_{z},+}^{(m)}(r, \theta, z)=e^{i k_{z} z} e^{-\alpha z} e^{i m \theta} J_{m}\left(k_{r} r\right)|+\rangle
$$

with

$$
k_{r}=\sqrt{\frac{m_{0}}{m_{1}}-k_{z}^{2}-\alpha^{2}} .
$$

If instead we adopt the condition (33), the corresponding values of $\alpha$ become

$$
\alpha=\frac{v \pm \sqrt{v^{2}-4 m_{1}\left(m_{0}-m_{1} k_{r}^{2}\right)}}{2 m_{1}},
$$

which implies a penetration length $1 / \alpha$ diverging at the line of nodes. The relation (35) only makes sense if $4 m_{0} m_{1}>v^{2}$, while in the regime $4 m_{0} m_{1}<v^{2}$ all the evanescent states are found using (36). As in the case of the Weyl semimetal, this disjunctive allows us to distinguish between two different classes of nodal-line semimetals, that we denote as type A (for $4 m_{0} m_{1}>v^{2}$ ) and type $\mathrm{B}$ (for $4 m_{0} m_{1}<v^{2}$ ). The type $\mathrm{A}$ corresponds to the regime that is expected to prevail for realistic materials providing examples of nodal-line semimetals. For example, most part of the $k \cdot p$ Hamiltonians of the $\mathrm{CaP}_{3}$ family of materials (without taking into account spin-orbit coupling) fall into that category [23]. From the physical point of view, the two classes $\mathrm{A}$ and $\mathrm{B}$ can be discerned by the different penetration of the drumhead surface states into the material, which shows for a slab practically the same behavior as represented in the case of the Weyl semimetal in Fig. 2.

From a formal point of view, the difference between type A and type B nodal-line semimetals lies in the distinctive complex structures that develop in the plane $\left(k_{z}, \alpha\right)$. In a type A nodal-line semimetal which has, for instance, a finite circular section at the boundary $z=0$, the values of $k_{r}$ become quantized, which turns into the consequent quantization of the momentum $k_{z}$. It can be seen that the allowed values of $k_{z}+i \alpha$ leading to evanescent states emerge then as exceptional points in the extension of the momentum $k_{z}$ to the complex plane, as represented in Fig. 7. Those can be characterized indeed as branch points in the spectrum of the Hamiltonian for complex momentum, leading to branch cuts that run down to homologous branch points with the reversed sign of $\alpha$.

We observe in Fig. 7 that the exceptional points can be grouped forming quartets, which is a consequence of

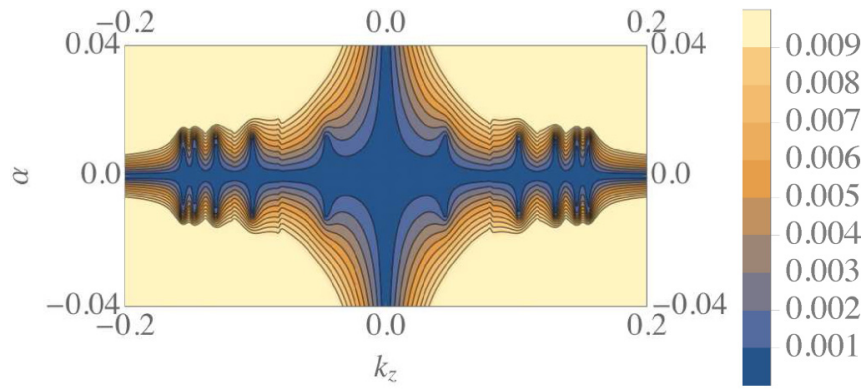

(a)

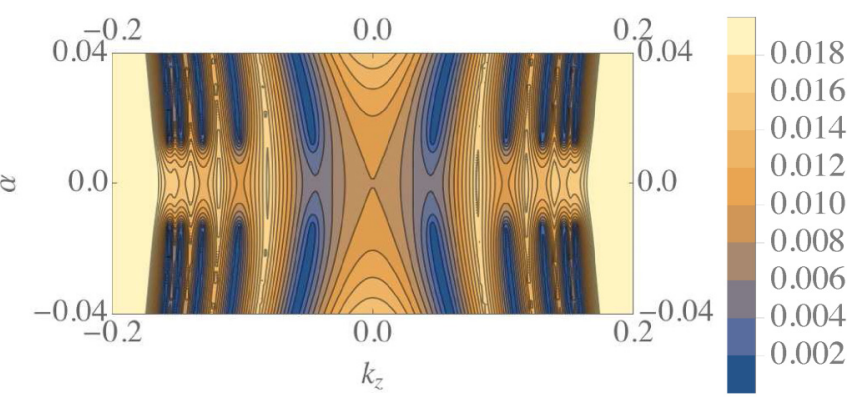

(b)

FIG. 7. Contour plots of the absolute value of the imaginary part (a) and the real part (b) of the lowest eigenvalue in the spectrum of $H_{\mathrm{NL}}$ for evanescent states with angular momentum $m=0$ in a semi-infinite cylindrical geometry with radius $R=100 a$ ( $a$ being the microscopic length scale in the model), for $m_{0}=0.1 \mathrm{eV}, m_{1} / a^{2}=4.0 \mathrm{eV}$, and $v / a=0.1 \mathrm{eV}$. The units are the same as in Fig. 4 .

the invariance of the Hamiltonian (21) under the operations defined by (19) and (20). We have actually

$$
\begin{aligned}
& {\left[H_{\mathrm{NL}}, I\right]=0,} \\
& {\left[H_{\mathrm{NL}}, T\right]=0 .}
\end{aligned}
$$

The invariance under $T$ is responsible for the fact that each pair of evanescent states with opposite sign of $k_{z}$ may have the same wave function at $z=0$. This symmetry is crucial in order to enforce the boundary conditions for the surface states at the edge of the semimetal, allowing to build for instance linear combinations of evanescent states that vanish at $z=0$. Moreover, the stability of the surface states is also guaranteed by the separation in the $\left(k_{z}, \alpha\right)$ plane between evanescent states related by the $I$ and $T$ operations, which lend topological protection as the branch cuts running between the respective branch points cannot be closed under small perturbations.

Such a nontrivial realization of the invariance under $I$ and $T$ holds only in the case of the type A nodal-line semimetal, as for type $\mathrm{B}$ all the evanescent states have $k_{z}=0$. In this latter case, they correspond to exceptional points in the spectrum of $H_{\mathrm{NL}}$ which fall along the imaginary axis in the $\left(k_{z}, \alpha\right)$ complex plane. A representation of the sequence of branch points for a type B nodal-line semimetal with cylindrical geometry is shown in Fig. 8. We notice that the plot has a series of discontinuities, which arise from the fact that the lowest eigenvalue of $H_{\mathrm{NL}}$ is found in different subbands as the 


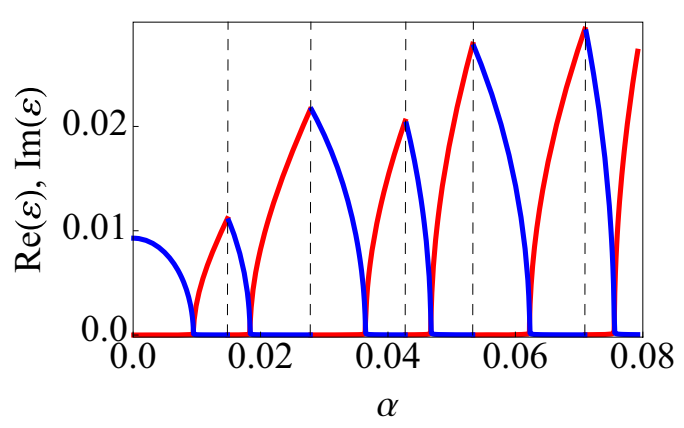

FIG. 8. Plot of the absolute value of the real part (blue lines) and the imaginary part (red lines) of the lowest eigenvalue in the spectrum of $H_{\mathrm{NL}}$ for evanescent states with angular momentum $m=$ 0 in a semi-infinite cylindrical geometry with radius $R=100 a$ ( $a$ being the microscopic length scale in the model), for $m_{0}=0.1 \mathrm{eV}$, $m_{1} / a^{2}=2.0 \mathrm{eV}$, and $v / a=1.0 \mathrm{eV}$. The units are the same as in Fig. 4. The dashed lines are used to separate the dispersion of different subbands, which correspond to the evolution of different eigenvalues in the diagonalization of $H_{\mathrm{NL}}$.

value of $\alpha$ increases. In each continuous region, we observe a clear characterization of the branch point as the location where the real and the imaginary part of the eigenvalue vanish.

The plot in Fig. 8 highlights an important property that applies both to type A and type B nodal-line semimetals. As already mentioned, it is clearly observed in the figure that exceptional points with different values of the complex momentum belong in general to different subbands in the spectrum of $H_{\mathrm{NL}}$. This can be also appreciated (though less neatly) in the representation of Fig. 7, where a careful inspection shows that a line of discontinuity exists in the contour plot between each two consecutive branch cuts. From the point of view of the complex structure, this means that exceptional points with different values of $k_{z}+i \alpha$ belong in general to different branches of the Riemann sheet arising from the diagonalization of $H_{\mathrm{NL}}$ for complex momentum. This implies that it is not possible in general to undo the branch cuts by merging contiguous exceptional points. The only branch points that can be made to coalesce are those that pertain to the same branch in the complex structure-which, in the case of a type A semimetal, are those connected precisely by the $I$ and $T$ operations. This reassures once more the topological stability of the collection of drumhead surface states, implied in this framework by the underlying complex structure of the spectrum.

\section{EVANESCENT STATES IN NODAL-LINE SEMIMETAL UNDER ELECTROMAGNETIC RADIATION}

A very interesting line of research is the control of quantum properties by external ac fields [24-29]. In this search, it has been found that the effect of electromagnetic radiation may change the properties of 2D semimetals, opening a gap in the bulk and leading to chiral currents at the boundary of the electron system [30-36]. The effect of the radiation has been also investigated in the case of 3D Dirac and Weyl semimetals, finding that a circularly polarized photon field has the ability to shift the Dirac or Weyl points in momentum space [37-39]. New surface states have been also discovered in Weyl semimetals illuminated by monochromatic radiation, forming bands with macroscopic degeneracy and rotating currents [17]. Another interesting result is that, using circularly polarized light in the proper direction, a nodal-line semimetal can be transformed into a Weyl semimetal [40-42].

In this section, we show that the idea of describing the surface states by exceptional points in the spectrum can be generalized to the case of the nodal-line semimetals under electromagnetic radiation. This is a relevant instance to check the topological protection that arises when extending the momenta to the complex plane, since the effects of the radiation can be studied in regimes where it is not a small perturbation.

The coupling to the vector potential can be done in the usual fashion, adopting the Peierls prescription $\mathbf{k} \rightarrow \mathbf{k}+\mathbf{A}$. In the case of circularly polarized radiation sent along the $z$ direction, we have

$$
\mathbf{A}=(A \cos (\Omega t), A \sin (\Omega t), 0) .
$$

The Hamiltonian becomes then

$$
\begin{aligned}
H= & \left(m_{0}+m_{1}\left(\partial_{x}^{2}+\partial_{y}^{2}+2 i A \cos (\Omega t) \partial_{x}\right.\right. \\
& \left.\left.+2 i A \sin (\Omega t) \partial_{y}-A^{2}+\partial_{z}^{2}\right)\right) \sigma_{z}-i v \partial_{z} \sigma_{x} \\
= & \left(m_{0}+m_{1}\left(\partial_{x}^{2}+\partial_{y}^{2}+i A e^{i \Omega t} \partial_{-}\right.\right. \\
& \left.\left.+i A e^{-i \Omega t} \partial_{+}-A^{2}+\partial_{z}^{2}\right)\right) \sigma_{z}-i v \partial_{z} \sigma_{x}
\end{aligned}
$$

with

$$
\begin{aligned}
& \partial_{-}=\partial_{x}-i \partial_{y}, \\
& \partial_{+}=\partial_{x}+i \partial_{y} .
\end{aligned}
$$

We have the commutation rules with the angular momentum $L_{z}=-i x \partial_{y}+i y \partial_{x}:$

$$
\begin{gathered}
{\left[L_{z}, \partial_{-}\right]=-\partial_{-},} \\
{\left[L_{z}, \partial_{+}\right]=\partial_{+} .}
\end{gathered}
$$

From these relations, it can be easily seen that

$$
\begin{gathered}
e^{i L_{z} \Omega t} \partial_{-} e^{-i L_{z} \Omega t}=e^{-i \Omega t} \partial_{-}, \\
e^{i L_{z} \Omega t} \partial_{+} e^{-i L_{z} \Omega t}=e^{i \Omega t} \partial_{+} .
\end{gathered}
$$

We can now rely on Eqs. (46) and (47) to pass to a timeindependent Hamiltonian by applying the unitary transformation

$$
U=e^{-i L_{z} \Omega t}
$$

We have

$$
\begin{aligned}
\widetilde{H}= & U^{\dagger} H U-i U^{\dagger} \partial_{t} U \\
= & \left(m_{0}+m_{1}\left(\partial_{x}^{2}+\partial_{y}^{2}+i A \partial_{-}+i A \partial_{+}-A^{2}+\partial_{z}^{2}\right)\right) \\
& \times \sigma_{z}-i v \partial_{z} \sigma_{x}-\Omega L_{z} .
\end{aligned}
$$

We can now perform an analysis of the evanescent states under radiation, following that accomplished before for the Hamiltonian $H_{\mathrm{NL}}$. The idea is to focus on evanescent states decaying as

$$
\chi(r, \theta, z) \sim e^{i k_{z} z} e^{-\alpha z} f(r, \theta)
$$


We look in particular for zero-energy modes, which must correspond to solutions of the equation

$$
\begin{aligned}
& {\left[m_{0}+m_{1}\left(\frac{1}{r} \frac{\partial}{\partial r}\left(r \frac{\partial}{\partial r}\right)+\frac{\partial_{\theta}^{2}}{r^{2}}+i A \partial_{-}+i A \partial_{+}-A^{2}\right.\right.} \\
& \left.\left.-k_{z}^{2}+\alpha^{2}-2 i k_{z} \alpha\right)\right] \sigma_{z} \chi_{k_{z}}+v\left(k_{z}+i \alpha\right) \sigma_{x} \chi_{k_{z}} \\
& \quad+i \Omega \partial_{\theta} \chi_{k_{z}}=0 .
\end{aligned}
$$

We perform first a numerical analysis of the problem, in which it is convenient to apply the gauge transformation

$$
\chi_{k_{z}}=e^{-i A x} \tilde{\chi}_{k_{z}} \text {. }
$$

This converts Eq. (51) into

$$
\begin{aligned}
& {\left[m_{0}+m_{1}\left(\frac{1}{r} \frac{\partial}{\partial r}\left(r \frac{\partial}{\partial r}\right)+\frac{\partial_{\theta}^{2}}{r^{2}}-k_{z}^{2}+\alpha^{2}-2 i k_{z} \alpha\right)\right]} \\
& \quad \times \sigma_{z} \tilde{\chi}_{k_{z}}+v\left(k_{z}+i \alpha\right) \sigma_{x} \tilde{\chi}_{k_{z}}+i \Omega \partial_{\theta} \tilde{\chi}_{k_{z}} \\
& \quad-A \Omega r \sin (\theta) \widetilde{\chi}_{k_{z}}=0
\end{aligned}
$$

Then one can check that it is possible to adjust the values of $k_{z}$ and $\alpha$ to obtain solutions of Eq. (53). The numerical resolution can be done for instance in a cylinder with $r<R$, in which there is a finite number of solutions depending on the radius $R$. These can be more easily visualized computing the spectrum of the operator in Eq. (53) in the complex plane $\left(k_{z}, \alpha\right)$, which leads in general to a picture like that represented in Fig. 9.

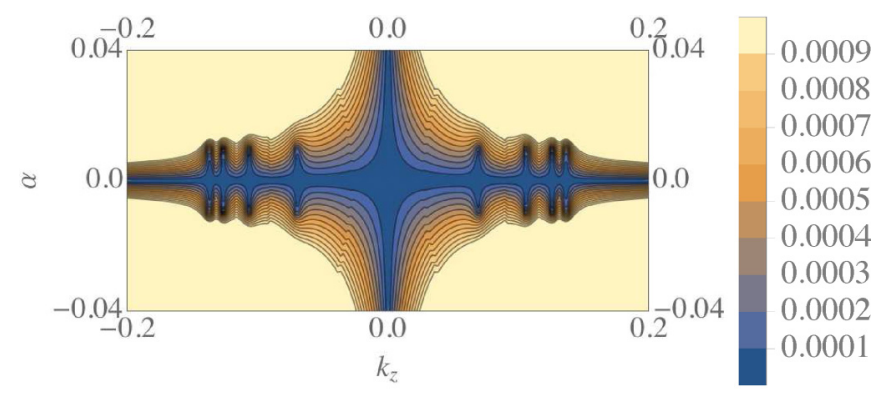

(a)

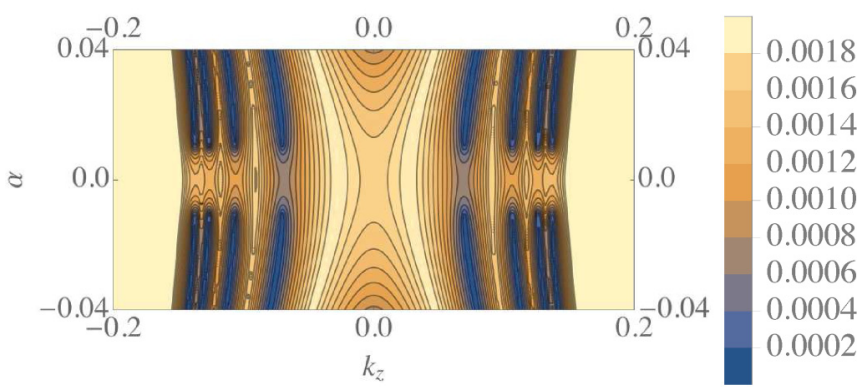

(b)

FIG. 9. Contour plots of the absolute value of the imaginary part (a) and the real part (b) of the lowest eigenvalue in the spectrum of $\widetilde{H}$ for evanescent states in a semi-infinite cylindrical geometry with radius $R=100 a$ ( $a$ being the microscopic length scale in the model) for $m_{0}=0.01 \mathrm{eV}, m_{1} / a^{2}=0.5 \mathrm{eV}, v / a=0.01 \mathrm{eV}, A a=0.05$, and $\Omega=1.0 \mathrm{eV}$. The units are the same as in Fig. 4 .
We observe that the zero-energy modes correspond once again to exceptional points in a spectrum of complex eigenvalues, with a structure similar to that already found in the absence of electromagnetic radiation. The exceptional points are easily identified as branch points with a tail where the lowest eigenvalue has zero imaginary part, and an opposite tail where the real part of the eigenvalue vanishes. This is the typical behavior for a square root singularity, which is also consistent with the structure of the branch cuts connecting homologous branch points across the $k_{z}$ axis, as seen in Fig. 9.

We can complement the numerical approach with an analytic search of the solutions of Eq. (51) when the surface has infinite size. We can start with the set of states spanned by the basis

$$
\begin{aligned}
& \chi_{k_{z},+}^{(m)}(r, \theta, z)=e^{i k_{z} z} e^{-\alpha z} e^{i m \theta} J_{m}\left(\hat{k}_{r} r\right)|+\rangle, \\
& \chi_{k_{z},-}^{(m)}(r, \theta, z)=e^{i k_{z} z} e^{-\alpha z} e^{i m \theta} J_{m}\left(\hat{k}_{r} r\right)|-\rangle,
\end{aligned}
$$

where

$$
\hat{k}_{r}=\sqrt{\frac{m_{0}}{m_{1}}-k_{z}^{2}+\alpha^{2}-A^{2}-\delta^{2}} .
$$

In this case, we keep $\alpha$ and $\delta$ as free parameters that we have to adjust in order to find the solutions of Eq. (51). This provides us with a very flexible collection of states, which proves to be large enough to capture the evanescent zero-energy modes.

The action of the off-diagonal perturbations induced in (51) by the radiation is given by

$$
\begin{gathered}
\partial_{-} \sigma_{z} \chi_{k_{z}, \pm}^{(m)}=\hat{k}_{r} \chi_{k_{z}, \mp}^{(m-1)}, \\
\partial_{+} \sigma_{z} \chi_{k_{z}, \pm}^{(m)}=-\hat{k}_{r} \chi_{k_{z}, \mp}^{(m+1)} .
\end{gathered}
$$

Then, the states we obtain by the action of $\widetilde{H}$ remain in the subspace we have defined:

$$
\begin{aligned}
\widetilde{H} \chi_{k_{z},+}^{(m)}= & i m_{1} A \hat{k}_{r} \chi_{k_{z},-}^{(m-1)}-i m_{1} A \hat{k}_{r} \chi_{k_{z},-}^{(m+1)}+m_{1} \delta^{2} \chi_{k_{z},-}^{(m)} \\
& -\left(v \alpha+i\left(2 m_{1} \alpha-v\right) k_{z}\right) \chi_{k_{z},-}^{(m)}-m \Omega \chi_{k_{z},+}^{(m)}, \\
\widetilde{H} \chi_{k_{z},-}^{(m)}= & i m_{1} A \hat{k}_{r} \chi_{k_{z},+}^{(m-1)}-i m_{1} A \hat{k}_{r} \chi_{k_{z},+}^{(m+1)}+m_{1} \delta^{2} \chi_{k_{z},+}^{(m)} \\
- & \left(-v \alpha+i\left(2 m_{1} \alpha+v\right) k_{z}\right) \chi_{k_{z},+}^{(m)}-m \Omega \chi_{k_{z},-}^{(m)} .
\end{aligned}
$$

One can check that, for not too large values of $k_{z}$ and $A$, it is possible to find a point in the space of parameters $(\alpha, \delta)$ where the eigenvalue of $\widetilde{H}$ becomes zero. This arises as a branch point in the spectrum of complex eigenvalues, as represented in Fig. 10. This procedure works up to certain limit values of $A$ and $k_{z}$, beyond which the branch point is lost and there is no signature of zero-energy modes. Such a limitation can be explained from the expression of the momentum $\hat{k}_{r}$ in (56). The parameters $\alpha$ and $\delta$ become only comparable to $A$ or $k_{z}$ when these are relatively small, which means that we have in general zero-energy modes for $m_{0} / m_{1} \gtrsim k_{z}^{2}+A^{2}$. Alternatively, we can express this constraint in terms of $\hat{k}_{r}$ as $m_{0} / m_{1} \gtrsim \hat{k}_{r}^{2}+A^{2}$, which may be seen as the requirement that the vector potential does not shift the momentum away from the nodal ring.

If we plot the spectrum as a function of $k_{z}+i \alpha$, once the appropriate value of $\delta$ is set, we get the image shown in Fig. 11. 


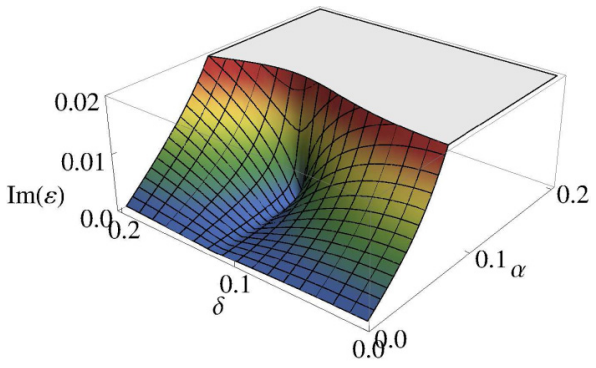

(a)

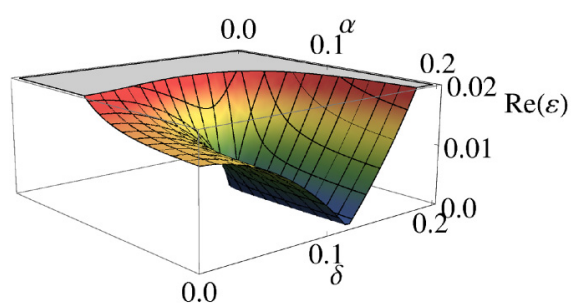

(b)

FIG. 10. Plot of the absolute value of the imaginary part (a) and the real part (b) of the lowest eigenvalue of $\widetilde{H}$ for evanescent states with variable parameters $\alpha$ and $\delta$, for $k_{z} a=0.2$ and $m_{0}=1.0 \mathrm{eV}$, $m_{1} / a^{2}=1.0 \mathrm{eV}, v / a=0.2 \mathrm{eV}, A a=0.1$, and $\Omega=0.5 \mathrm{eV}$. The units are the same as in Fig. 4.

We observe there a line of zero-energy modes, which may be thought as the accumulation of the exceptional points already found in the finite-size numerical approach. This shows that a continuum of evanescent modes persist under the effect of the electromagnetic radiation, arising as a result of the hybridiza-

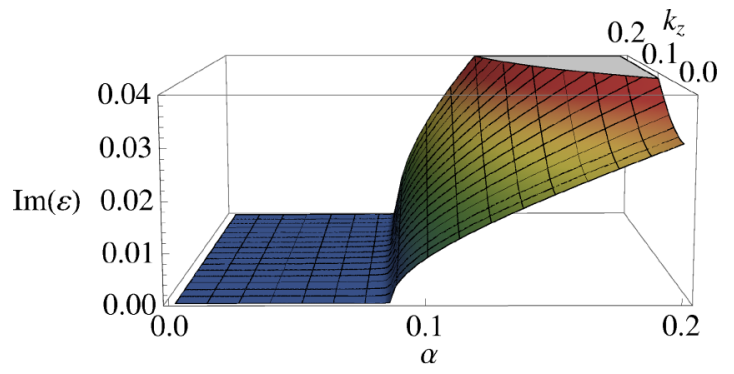

(a)

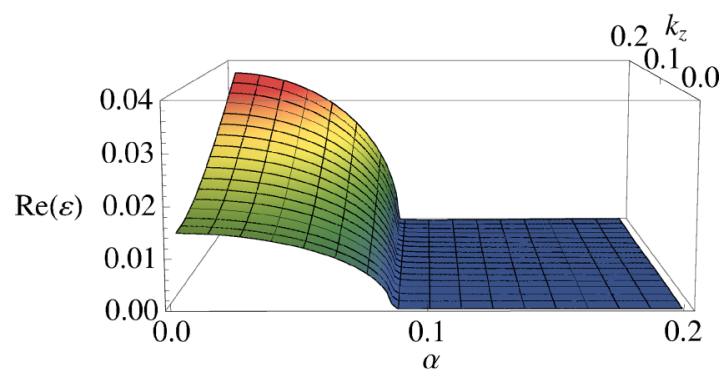

(b)

FIG. 11. Plot of the absolute value of the imaginary part (a) and the real part (b) of the lowest eigenvalue of $\widetilde{H}$ for evanescent states with complex momentum $k_{z}+i \alpha$, for $m_{0}=1.0 \mathrm{eV}, m_{1} / a^{2}=1.0 \mathrm{eV}$, $v / a=0.2 \mathrm{eV}, A a=0.1$, and $\Omega=0.5 \mathrm{eV}$. The units are the same as in Fig. 4. tion of drumhead states with different angular momentum and being labeled by the component $k_{z}$ of the momentum.

We have to remark, however, that, in the present case, the exceptional points we have found do not imply the existence of zero-energy surface states attached to a given boundary of the nodal-line semimetal. This is so as the evanescent eigenstates of $\widetilde{H}$ do not realize the symmetry required to build appropriate linear combinations that may guarantee the vanishing of the surface states at the boundary, say at $z=0$. In the case of the unperturbed nodal-line semimetal, we saw above that the crucial symmetry for the existence of zero-energy surface states was given by the $T$ operation in (20). The introduction of the radiation field breaks the time-reversal invariance, which explains that the evanescent modes corresponding to momenta $k_{z}$ and $-k_{z}$ have different wave functions at $z=0$ in the presence of the radiation. This stresses the significance of the symmetry of the Hamiltonian to guarantee the stability of the zero-energy surface states, together with the topological protection already provided by the existence of the exceptional points in the spectrum.

\section{CONCLUSIONS}

We have shown how the surface states in topological semimetals are associated to exceptional points of the spectrum in the extension of the Brillouin zone to complex values of momenta. These exceptional points are very robust under perturbations of the Hamiltonian as they can be only annihilated by merging them in pairs to close the branch cuts in the spectrum. This draws a useful way of understanding the topological protection of evanescent states like those forming the Fermi arcs in Weyl semimetals or the drumhead states in nodal-line semimetals. In this regard, the mechanism of topological protection seems to be rather different to that of the nodes of the semimetals themselves (and different also to that studied recently in the case of 3D Dirac semimetals [14]).

We have also shown that the evanescent states decay exponentially into the semimetal, and we have illustrated the dependence of the penetration length according to the parameters of a model Hamiltonian for both Weyl and nodalline semimetals. The penetration into the semimetal can be very short and oscillating, or longer and with pure exponential decay, depending on the ratio between the linear and the quadratic terms in the Hamiltonian. According to this ratio, we have classified the topological semimetals as type A or type B. In general we expect the quadratic terms in the model to dominate and, thus, the type A (the short penetration with oscillations) to be more common in real materials. However, at least in the case of $\mathrm{Na}_{3} \mathrm{Bi}$, a Dirac semimetal, the model parameters computed through DFT simulations imply a type $\mathrm{B}$ behavior, mainly due to the small absolute value of the mass parameter $M_{0}$ [43]. On the contrary, other published parameter values lead to type A behavior, like in the Dirac and Weyl semimetal family of $X Y \mathrm{Bi}(X=\mathrm{Ba}, \mathrm{Eu} ; Y=\mathrm{Cu}, \mathrm{Ag}$, and $\mathrm{Au})$ [44]. Also, in the proposals for artificial topological semimetals in photonic or cold atom setups, both regimes could easily be reached [45-48]. The distinction between type A and type B is also formally related to the different way in which the branch cuts corresponding to the exceptional points are arranged in the complex plane. 
An important consequence of the shape of the evanescent wave functions is that, for type B semimetals with pure exponential decay of the surface states, it is not possible to fulfill slab boundary conditions with a linear combination of states on just one of the surfaces. In this case, surface states of different chiralities will be coupled in the two surfaces of the slab. This coupling will depend on the ratio between the decay length of the evanescent waves and the total width of the slab. On the contrary, in the case of type A topological semimetals with oscillatory decay, it is possible to construct surface states in one of the surfaces fulfilling the boundary conditions of the slab. We expect this difference to have very important consequences allowing to distinguish experimentally both scenarios. Any local probe in one of the surfaces affecting only surface states will have no effect on the opposite surface for type A semimetals, while it will affect the other surface for type B semimetals. A full evaluation of the implications of these properties for experiments and applications is out of the scope of this paper, but we expect that setups probing tunneling, like two terminal conductance measurements, will be qualitatively different between slabs of relatively small widths $(\sim 100 \mathrm{~nm})$ of type A and type B semimetals.

Besides, a more direct way of measuring these differences between the surface states may be to explore how different properties evolve as a function of the width of a thin film, as has been done recently for the topological surface states of antimony [49]. For type B topological semimetals, we expect a monotonous dependence of the properties of the surface states as a function of the width of the thin film, while for type A topological semimetals a non-monotonous dependence has to be found. In particular, quasiparticle interference patterns can be studied. They will show inter-surface coupling that will depend nonmonotonously on the width of the film in the case of type A topological semimetals. The energy dependence can be used to extract the gap opened at the Fermi arcs due to the finite width of the film. This gap is, in general, larger and monotonous as a function of the width in type B semimetals and smaller and showing deep minima for particular values of the width in type A semimetals, as shown in Fig. 3.

In nodal-line semimetals, we have also studied the effect of an external ac field on the surface states. Evanescent states with different angular momentum are mixed by the external field. Interestingly, the resulting states are still in correspondence to exceptional points in the complex plane and they are protected from small perturbations as such. These mixtures of states with different angular momenta should carry a rotating current similarly to that studied before in Weyl semimetals [17]. However, the reduction of symmetry from the ac field implies that the zero-energy surface states of nodal-line semimetals are in general not stable in the presence of radiation, as this breaks the required invariance to comply with appropriate boundary conditions.

We believe our work paves the way for an alternative understanding of topological protection of edge states in gapless systems, based on the extension of the band structure for complex values of the momenta. In this complex momentum space, the surface states arise as exceptional points, making possible to study their stability by discerning the perturbations capable to close the branch cuts in the complex band structure. It would be an interesting future avenue to investigate the connection between this description of surface states and the topological properties of photonic materials or open quantum systems that can be described by actual non-Hermitian Hamiltonians [50-54].

\section{ACKNOWLEDGMENT}

We acknowledge financial support through Spanish Grants MINECO/FEDER No. FIS2015-63770-P and No. FIS201457432-P.
[1] M. Z. Hasan and C. L. Kane, Topological insulators, Rev. Mod. Phys. 82, 3045 (2010).

[2] X.-L. Qi and S.-C. Zhang, Topological insulators and superconductors, Rev. Mod. Phys. 83, 1057 (2011).

[3] G. Burns, Solid State Physics (Academic Press, New York, 1985).

[4] K. S. Novoselov, A. K. Geim, S. V. Morozov, D. Jiang, Y. Zhang, S. V. Dubonos, I. V. Grigorieva, and A. A. Firsov, Electric field effect in atomically thin carbon films, Science 306, 666 (2004).

[5] Z. K. Liu, B. Zhou, Y. Zhang, Z. J. Wang, H. M. Weng, D. Prabhakaran, S.-K. Mo, Z. X. Shen, Z. Fang, X. Dai, Z. Hussain and Y. L. Chen, Discovery of a three-dimensional topological dirac semimetal, $\mathrm{Na}_{3} \mathrm{Bi}$, Science 343, 864 (2014).

[6] M. Neupane, S.-Y. Xu, R. Sankar, N. Alidoust, G. Bian, C. Liu, I. Belopolski, T.-R. Chang, H.-T. Jeng, H. Lin, A. Bansil, F. Chou, and M. Z. Hasan, Observation of a three-dimensional topological Dirac semimetal phase in high-mobility $\mathrm{Cd}_{3} \mathrm{As}_{2}$, Nat. Commun. 5, 3786 (2014).

[7] S. Borisenko, Q. Gibson, D. Evtushinsky, V. Zabolotnyy, B. Büchner, and R. J. Cava, Experimental Realization of a ThreeDimensional Dirac Semimetal, Phys. Rev. Lett. 113, 027603 (2014).
[8] S.-Y. Xu, I. Belopolski, N. Alidoust, M. Neupane, G. Bian, C. Zhang, R. Sankar, G. Chang, Z. Yuan, C.-C. Lee, S.-M. Huang, H. Zheng, J. Ma, D. S. Sanchez, B. Wang, A. Bansil, F. Chou, P. P. Shibayev, H. Lin, S. Jia, and M. Z. Hasan, Discovery of a Weyl fermion semimetal and topological Fermi arcs, Science 349, 613 (2015).

[9] A. A. Burkov, Topological semimetals, Nat. Mater. 15, 1145 (2016).

[10] M. Phillips and V. Aji, Tunable line node semimetals, Phys. Rev. B 90, 115111 (2014).

[11] C. Chiu and A. Schnyder, Classification of reflection symmetry protected topological semimetals and nodal superconductors, Phy. Rev. B 90, 205136 (2014).

[12] X. Wan, A. M. Turner, A. Vishwanath, and S. Y. Savrasov, Topological semimetal and fermi-arc surface states in the electronic structure of pyrochlore iridates, Phys. Rev. B 83, 205101 (2011).

[13] K. Y. Yang, Y. M. Lu, and Y. Ran, Quantum Hall effects in a Weyl semimetal: Possible application in pyrochlores iridates, Phys. Rev. B 84, 075129 (2011).

[14] M. Kargarina, M. Randeria, and Y.-M. Lu, Are the surface Fermi arcs in Dirac semimetals topologically protected? PNAS 113, 8648 (2016). 
[15] E. V. Gorbar, V. A. Miransky, I. A. Shovkovy, and P. O. Sukhachov, Surface Fermi arcs in $\mathbb{Z}_{2}$ Weyl semimetals $A_{3} \mathrm{Bi}$ ( $A=\mathrm{Na}, \mathrm{K}, \mathrm{Rb}$ ), Phys. Rev. B 91, 235138 (2015); Dirac semimetals $A_{3} \mathrm{Bi}(A=\mathrm{Na}, \mathrm{K}, \mathrm{Rb})$ as $\mathbb{Z}_{2}$ Weyl semimetals, 91, 121101(R) (2015).

[16] S. Tchoumakov, M. Civelli, and M. O. Goerbig, Magnetic description of the Fermi arc in type-I and type-II Weyl semimetals, Phys. Rev. B 95, 125306 (2017).

[17] J. González and R. A. Molina, Macroscopic Degeneracy of ZeroMode Rotating Surface States in 3D Dirac and Weyl Semimetals Under Radiation, Phys. Rev. Lett. 116, 156803 (2016).

[18] Y. Xu, S.-T. Wang, and L.-M. Duan, Weyl Exceptional Rings in a Three-Dimensional Dissipative Cold Atomic Gas, Phys. Rev. Lett. 118, 045701 (2017).

[19] A. A. Soluyanov, D. Gresch, Z. Wang, Q. Wu, M. Troyer, X. Dai, and B. A. Bernevig, Type-II Weyl semimetals, Nature 527, 495 (2015).

[20] Y. Xu, F. Zhang, and C. Zhang, Structured Weyl Points in Spin-Orbit Coupled Fermionic Superfluids, Phys. Rev. Lett. 115, 265304 (2015).

[21] M. V. Berry, Physics of non-Hermitian degeneracies, Czech. J. Phys. 54, 1039 (2004).

[22] W. D. Heiss, The physics of exceptional points, J. Phys. A: Math. Theor. 45, 444016 (2012).

[23] Q. Xu, R. Yu, Z. Fang, X. Dai, and H. Weng, Topological nodal line semimetals in the $\mathrm{CaP}_{3}$ family of materials, Phys. Rev. B 95, 045136 (2017).

[24] D. H. Dunlap and V. M. Kenkre, Dynamic localization of a charged particle moving under the influence of an electric field, Phys. Rev. B 34, 3625 (1986).

[25] F. Grossmann, T. Dittrich, P. Jung, and P. Hänggi, Coherent Destruction of Tunneling, Phys. Rev. Lett. 67, 516 (1991).

[26] M. Holthaus, G. H. Ristow, and D. W. Hone, ac-Field-Controlled Anderson Localization in Disordered Semiconductor Superlattices, Phys. Rev. Lett. 75, 3914 (1995).

[27] G. Platero and R. Aguado, Photon-assisted transport in semiconductor nanostructures, Phys. Rep. 395, 1 (2004).

[28] D. F. Martinez and R. A. Molina, Delocalization induced by low-frequency driving in disordered tight-binding lattices, Phys. Rev. B 73, 073104 (2006).

[29] C. E. Creffield, Quantum Control and Entanglement Using Periodic Driving Fields, Phys. Rev. Lett. 99, 110501 (2007).

[30] T. Oka and H. Aoki, Photovoltaic Hall effect in graphene, Phys. Rev. B 79, 081406 (2009).

[31] N. H. Lindner, G. Refael, and V. Galitski, Floquet topological insulators, Nat. Phys. 7, 490 (2011).

[32] J.-I. Inoue and A. Tanaka, Photoinduced Transition between Conventional and Topological Insulators in Two-Dimensional Electronic Systems, Phys. Rev. Lett. 105, 017401 (2010).

[33] T. Kitagawa, E. Berg, M. Rudner, and E. Demler, Topological characterization of periodically driven quantum systems, Phys. Rev. B 82, 235114 (2010).

[34] T. Kitagawa, T. Oka, A. Brataas, L. Fu, and E. Demler, Transport properties of nonequilibrium systems under the application of light: Photoinduced quantum Hall insulators without Landau levels, Phys. Rev. B 84, 235108 (2011).

[35] Z. Gu, H. A. Fertig, D. P. Arovas, and A. Auerbach, Floquet Spectrum and Transport through an Irradiated Graphene Ribbon, Phys. Rev. Lett. 107, 216601 (2011).
[36] A. Gómez-León and G. Platero, Floquet-Bloch Theory and Topology in Periodically-Driven Lattices, Phys. Rev. Lett. 110, 200403 (2013).

[37] R. Wang, B. Wang, R. Shen, L. Sheng, and D. Y. Xing, Floquet Weyl semimetal induced by off-resonant light, Europhys. Lett. 105, 17004 (2014).

[38] A. Narayan, Floquet dynamics in two-dimensional semi-Dirac semimetals and three-dimensional Dirac semimetals, Phys. Rev. B 91, 205445 (2015).

[39] C.-K. Chan, P. A. Lee, K. S. Burch, J. H. Han, and Y. Ran, When Chiral Photons Meet Chiral Fermions - Photoinduced Anomalous Hall Effects in Weyl Semimetals, Phys. Rev. Lett. 116, 026805 (2016).

[40] Z. Yan and Z. Wang, Tunable Weyl Points in Periodically Driven Nodal Line Semimetals, Phys. Rev. Lett. 117, 087402 (2016).

[41] A. Narayan, Tunable point nodes from line-node semimetals via application of light, Phys. Rev. B 94, 041409(R) (2016).

[42] C.-K. Chan, Y.-T. Oh, J. H. Han, and P. A. Lee, Type-II Weyl cone transitions in driven semimetals, Phys. Rev. B 94, 121106(R) (2016).

[43] Z. Wang, Y. Sun, X.-Q. Chen, C. Franchini, G. Xu, H. Weng, $X$. Dai, and Z. Fang, Dirac semimetal and topological phase transitions in $\mathrm{A}_{3} \mathrm{Bi}(\mathrm{A}=\mathrm{Na}, \mathrm{K}, \mathrm{Rb})$, Phys. Rev. B 85, 195313 (2012).

[44] Y. Du, B. Wan, D. Wang, L. Sheng, C.-G. Duan, and X. Wan, Dirac and Weyl semimetal in $\mathrm{XYBi}(\mathrm{X}=\mathrm{Ba}, \mathrm{Eu} ; \mathrm{Y}=\mathrm{Cu}, \mathrm{Ag}$ and Au), Sci. Rep. 5, 14423 (2015).

[45] T. Dubcek, C. J. Kennedy, L. Lu, W. Ketterlee, M. Soljačić, and H. Buljan, Weyl Points in Three-Dimensional Optical Lattices: Synthetic Magnetic Monopoles in Momentum Space, Phys. Rev. Lett. 114, 225301 (2015).

[46] Y. Xu and C. Zhang, Dirac and Weyl rings in three-dimensional cold-atom optical lattices, Phys. Rev. A 93, 063606 (2016).

[47] L. Lu, L. Fu, J. D. Joannopoulos, and M. Soljačić, Weyl points and line nodes in gyroid photonic crystals, Nat. Photonics 7, 294 (2013).

[48] L. Lu, Z. Wang, D. Ye, L. Ran, L. Fu, J. D. Joannopoulos, and M. Soljačić, Experimental observation of Weyl points, Science 349, 622 (2015).

[49] G. Yao, Z. Luo, W. Xu, Y. P. Feng, and X. Wang, Evolution of topological surface states in antimony ultra-thin films, Sci. Rep. 3, 02010 (2013).

[50] K. Esaki, M. Sato, K. Hasebe, and M. Kohmoto, Edge states and topological phases in non-Hermitian systems, Phys. Rev. B 84, 205128 (2011).

[51] S. Diehl, E. Rico, M. A. Baranov, and P. Zoller, Topology by dissipation in atomic quantum wires, Nat. Phys. 7, 971 (2011).

[52] J. M. Zeuner, M. C. Rechtsman, Y. Plotnik, Y. Lumer, S. Nolte, M. S. Rudner, M. Segev, and A. Szameit, Observation of a Topological Transition in the Bulk of a Non-Hermitian System, Phys. Rev. Lett. 115, 040402 (2015).

[53] P. San-José, J. Cayao, E. Prado, and R. Aguado, Majorana bound states from exceptional points in non-topological superconductors, Sci. Rep. 6, 21427 (2016).

[54] B. Peng, S. K. Özdemir, M. Liertzer, W. Chen, J. Kramer, H. Yilmaz, J. Wiersig, S. Rotter, and L. Yang, Chiral modes and directional lasing at exceptional points, Proc. Natl. Acad. Sci. USA 113, 6845 (2016). 